\title{
High-SNR Staining Algorithm for One-Way Wave Equation-Based Modelling and Imaging
}

\author{
Yaxing $\mathrm{Li}^{1,2}$ and Xiaofeng Jia ${ }^{1,2, *}$ \\ ${ }^{1}$ Laboratory of Seismology and Physics of Earth's Interior, School of Earth and Space \\ Sciences, University of Science and Technology of China, Hefei 230026, China. \\ 2 National Geophysical Observatory at Mengcheng, University of Science and \\ Technology of China, Auhui, China.
}

Received 30 April 2018; Accepted (in revised version) 11 October 2018

\begin{abstract}
Staining algorithms based on two-way wave equation migration methods have been applied to improve the signal-to-noise ratio (SNR) of poorly illuminated structures such as those in subsalt zones. In regular staining algorithms, when a source wavefield reaches the stained area that is associated with the target structures, a new wavefield called stained wavefield is excited, and this stained wavefield forward extrapolates synchronously with the real source wavefield. The forward-extrapolated stained and real source wavefields are cross-correlated with the backward-extrapolated receiver wavefield, and we obtain the stained and the real reverse time migration (RTM) images. The staining algorithms for RTM can suppress the noise of non-target regions and obtain high SNR images of the target structures. Whereas RTM methods are limited by the low computational efficiency and SNR, by contrast, one-way wave equation migration (OWEM) methods have the advantages of high efficiency and no interference from multiples. Thus, we developed a new staining method based on the generalised screen propagator (GSP) as a case of OWEM methods for subsalt imaging. Furthermore, a new stained wavefield called stained receiver wavefield is proposed here, forming two new staining strategies for seismic imaging, in which forwardpropagated source and backward-propagated receiver wavefields can be conveniently selected to be stained at the stained area. Numerical experiments demonstrated that this staining GSP method is more effective in improving the SNR of subsalt structures compared to conventional GSP migration and RTM methods; moreover, these new staining strategies as applied to the OWEM methods can greatly improve the SNR of weakly illuminated structures in subsalt zones, in comparison with regular staining algorithms for one-way methods.
\end{abstract}

AMS subject classifications: 86-08, 86A15, 86A20, 86A22

Key words: One-way, two-way, modelling, imaging, staining, RTM.

*Corresponding author. Email addresses: yxli2017@mail.ustc.edu.cn (Y. X. Li), xjia@ustc.edu.cn (X. F. Jia) 


\section{Introduction}

Seismic imaging is a technology that returns reflected and diffracted waves to the subsurface where they are generated, allowing images of the underground structures to be obtained. However, weakly illuminated structures exist owing to the limited acquisition geometry, complex overburden structures, and reflector dip angles [59]. Subsalt basins are typical weakly illuminated structures owing to the defocusing by the salt boundaries and by the transmission and reflection losses induced by the strong velocity contrast between the salt and its surrounding sediments when seismic waves pass through the salt bodies $[23,32,35,39]$. Some approaches have been proposed to improve the illumination of subsalt shadow zones. O'Brien and Gray [40] put forward some proposals to improve the images of the structures below the salt by interpreting poor quality data and building accurate velocity models. Kessinger and Ramaswamy [29] applied mode converted energy to the depth migration for subsalt imaging. Some other studies have focused on compensating the illumination of subsalt regions [43,63], acquisition aperture correction $[4,55,56]$, and velocity model building $[24,33,48]$. There are many methods that have been developed for subsalt imaging by using multiples [11, 17, 35], turning waves [18], converted waves $[36,41,53,58]$, or vertical seismic profile data [3,44]. Moreover, Tang and Biondi [47] presented a strategy based on the target-oriented wavefield tomography using synthesised Born data and obtained improved SNR images of the subsalt structures. Yan et al. [62] presented a hybrid elastic one-way propagator for subsalt imaging. Although these efforts have made great progress in subsalt imaging, there are still problems in enhancing the illumination of the subsalt.

Chen and Jia [6] proposed a staining algorithm derived from the fate mapping technology in developmental biology for improving the SNR of poorly illuminated structures. In fate mapping, embryologists use 'vital dyes' to stain and label an undifferentiated embryonic cell, and then the stained cell goes through differentiation and development and can be seen in the adult organisms $[10,15]$. This technique is carried out for tracing the embryonic origin of various tissues in the adult organisms, which establishes a link between a single cell or tissue at a certain stage and adult cells at later stages of development. Corresponding to the fate mapping method, in staining algorithms, we first stain the target-related structures as a stained area, and when the source wavefield reaches the stained area, a new wavefield called stained wavefield is excited. This stained wavefield, which is regarded as a virtual wavefield, propagates synchronously with the real source wavefield. Because the stained wavefield removes information unrelated to the target structures, we can use the stained wavefield for obtaining high SNR images of the target structures. Chen and Jia [5] applied the staining algorithm to broadband seismic illumination and resolution analysis. To overcome the problems of amplitude and waveform distortions, $\mathrm{Li}$ and Jia [34] proposed a new amplitude-preserved staining algorithm called generalised staining algorithm for seismic modelling and imaging. In addition, Jia and Yang [26] developed a memory-efficient staining algorithm for 3D seismic modelling and imaging. All the above staining algorithms are based on two-way 
wave equation methods.

Compared to the two-way wave-equation methods, OWEM methods that have been widely used in the industry have the advantages of high computational efficiency and no interference from the multiples. Claerbout [8] applied the finite-difference scheme to seismic migration and proposed a time-space $(t-x)$ domain OWEM algorithm based on the paraxial approximation to the wave equation. It is difficult for the OWEM methods to image the steep-dip structures and the media with strong lateral velocity contrasts, such as the salt bodies, because one-way wave propagators can hardly deal with largeangle wave propagation $[12,42,46]$. A large number of methods have been proposed to handle the difficulties of the one-way migration, for instance, high-order approximations $[1,16,31]$, phase-shift methods $[13,14,38]$, and dual-domain methods [7, 20-22, 30, 42, 45]. $\mathrm{Wu}$ [50] summarised the developments and characteristics of various dual-domain methods and their applications. Many generalised screen propagators (GSPs) composed of pseudo-screen [21, 27, 28, 54, 57], Padé-screen [21, 60], and higher-order generalised screen $[30,37]$ have been developed for their high resolution, fidelity, and efficiency [50]. Jia and $\mathrm{Wu}$ [25] proposed a superwide-angle wavefront reconstruction method based on the GSP, which can deal with the large-angle propagations of wavefields. Other studies have been conducted to develop true-amplitude imaging methods based on the OWEM method $[64,65]$.

In this study, we developed a new staining method based on the OWEM methods to improve the SNR of subsalt structures, and we employed the Padé GSP as a case of oneway methods [60]. We used the new staining method to image two-dimensional (2D) subsalt structures and compared the results with those of conventional GSP and reverse time migration (RTM) methods. Moreover, we extended the construction of the stained wavefield from the source side to the receiver side, and the virtual receiver wavefield backward propagates synchronously with the real receiver wavefield. Two new staining strategies are proposed for imaging subsalt structures by using the stained receiver wavefield. One strategy is to cross-correlate the real source wavefield with the stained receiver wavefield; the other is to carry out the cross-correlation on the stained source and receiver wavefields; thus, we can totally obtain three stained images of the target structures.

\section{Theory}

\subsection{Theory of one-way wave equation migration}

On the basis of perturbation theory, the seismic wave velocity $v$ in the media can be divided into constant reference velocity $v_{0}$ and the velocity perturbation $\delta_{v}=v-v_{0}$, and the $2 \mathrm{D}$ acoustic wave equation of the frequency domain is defined as [60]

$$
\left(\frac{\partial^{2}}{\partial x^{2}}+\frac{\partial^{2}}{\partial z^{2}}+k_{0}^{2} n^{2}\right) u(x, z)=0
$$


where $u(x, z)$ refers to the wavefield, $k_{0}=\omega / v_{0}$ refers to the reference wavenumber, and $n=v_{0} / v$ refers to the refractive index. By factoring Eq. (2.1), we can obtain one-way wave equations for down- and up-going waves, given by

$$
\begin{aligned}
& \left(\frac{\partial}{\partial z}+i \sqrt{k_{0}^{2} n^{2}+\frac{\partial^{2}}{\partial x^{2}}}\right) u_{s}(x, z)=0, \\
& \left(\frac{\partial}{\partial z}-i \sqrt{k_{0}^{2} n^{2}+\frac{\partial^{2}}{\partial x^{2}}}\right) u_{r}(x, z)=0 .
\end{aligned}
$$

The OWEM method is based on the source wavefield $u_{s}(x, z)$ forward extrapolation and on the receiver wavefield $u_{r}(x, z)$ backward extrapolation in the depth direction.

GSPs as a dual domain one-way propagator have the features of relatively high resolution and fidelity in amplitude [50]. There are many expressions of GSPs derived from various approximations $[21,27,28,37,51,52,57,60]$. The expressions of GSPs derived from the first Padé approximation are characterised by $[19,60]$

$$
\begin{aligned}
& u_{s}\left(x, z_{k}+\Delta z\right)=\left(1+i \Delta z \frac{A}{k_{0}} \frac{\partial^{2}}{\partial x^{2}}\right) \cdot F^{-1} e^{i \Delta z k_{z}} F e^{-i \Delta z k_{0} \frac{\delta v\left(x, z_{k}\right)}{v\left(x, z_{k}\right)}} u_{s}\left(x, z_{k}\right), \\
& u_{r}\left(x, z_{k}+\Delta z\right)=\left(1-i \Delta z \frac{A}{k_{0}} \frac{\partial^{2}}{\partial x^{2}}\right) \cdot F^{-1} e^{i \Delta z k_{z}} F e^{-i \Delta z k_{0} \frac{\delta v\left(x, z_{k}\right)}{v\left(x, z_{k}\right)}} u_{r}\left(x, z_{k}\right),
\end{aligned}
$$

where $z_{k}$ is a specified depth; $F$ refers to the Fourier transform from the space domain to the wavenumber domain, while $F^{-1}$ refers to the inverse Fourier transform from the wavenumber domain to the space domain, and $A=(1 / 2)[F(1 / n)-1]$.

\subsection{Staining algorithms for OWEM}

Staining algorithms based on the RTM method have been used for tracing the propagation of the source wavefield in a particular target region and for improving the SNR of poorly illuminated structures in seismic imaging. In this work, we developed a new staining method based on the OWEM method for subsalt imaging. We constructed a spatial function to describe the stained area near the target structure. When the source wavefield propagates to the stained area, a new wavefield called stained source wavefield is excited, and this stained source wavefield at the stained area is regarded as the boundary condition and propagates synchronously with the real source wavefield. The stained source wavefield $\widetilde{u_{s}}(x, z)$ has the form of

$$
\left(\frac{\partial}{\partial z}+i \sqrt{k_{0}^{2} n^{2}+\frac{\partial^{2}}{\partial x^{2}}}\right) \widetilde{u}_{s}(x, z)=0,
$$

and the boundary condition of Eq. (2.6) is given by

$$
\left.\widetilde{u}_{s}(x, z)\right|_{D_{s}(x, z)=1}=u_{s}(x, z),
$$


where $D_{s}(x, z)=1$ and $D_{s}(x, z)=0$ are defined as the stained and the non-stained area, respectively. Eqs. (2.2) and (2.6) show that the real and the stained source wavefields propagate in the same velocity model; however, the propagations of the two wavefields are independent. This is the only connection between the stained and the real source wavefields at the stained area (i.e., Eq. (2.7)).

In fact, the propagation of the stained source wavefield is a process of reconstructing the target-oriented source wavefield. Next, we demonstrate the relationship between the stained and the real source wavefields and explain how to select the stained area in detail. The Huygens-Fresnel principle shows that, when the real source wavefield propagates to the stained area, the stained source wavefield is excited as a secondary wavefield and the disturbance of any point $M\left(x_{m}\right)$ (see Fig. 1) in the stained source wavefield is the coherent superposition of these secondary stained waves at a given point. According to the Kirchhoff integral of 2D wave equation, the forward-propagated real source wavefield in the frequency domain $u_{s}\left(x_{m}, \omega\right)$ of $M\left(x_{m}\right)$ inside the surface $\alpha$ is written as [2]

$$
u_{s}\left(x_{m}, \omega\right)=\int_{l=l_{1}+l_{2}}\left[\frac{\partial G\left(\boldsymbol{x}, x_{m}, \omega\right)}{\partial n} u_{s}(\boldsymbol{x}, \omega)-G\left(\boldsymbol{x}, \boldsymbol{x}_{m}, \omega\right) \frac{\partial u_{s}(\boldsymbol{x}, \omega)}{\partial n}\right] d l,
$$

where $G\left(x, x_{m}, \omega\right)$ and $u_{s}\left(x, x_{m}, \omega\right)$ are the Green function and the real source wavefield on the line $l$, respectively. $l=l_{1}+l_{2}$ is a closed line that contains the real source wavefield within the surface $\alpha$, and $l_{1}$ can be defined as a reflection-free boundary. $n$ represents the normal of the $l$ line (see Fig. 1(a)). We assume that $x$ goes to infinity along the line $l_{1}$, so the integral along $l_{2}$ in Eq. (2.8) can be ignored, and we can get the real source wavefield, given by [2]

$$
u_{s}\left(x_{m}, \omega\right)=\int_{l_{1}}\left[\frac{\partial G\left(x, x_{m}, \omega\right)}{\partial n} u_{s}(x, \omega)-G\left(x, x_{m}, \omega\right) \frac{\partial u_{s}(x, \omega)}{\partial n}\right] d l_{1} .
$$

When the stained area $D_{S}$ is selected, we can fully apply Eqs. (2.8) and (2.9) to the forwardextrapolated stained source wavefield. Hence, the staining algorithm is effective in reconstructing the target-oriented source wavefield. The problem is how we choose the stained area to improve the SNR of the target structure. If we choose the lower non-closed line $l_{2}$ as the stained area $\left(D_{s}=l_{2}\right)$ (see Fig. $1(\mathrm{~b})$ ), the forward-extrapolated real and stained source wavefields propagate downward according to the one-way wave equation, such that the stained source wavefield has no energy above the stained area and the stained wavefield at point $M$ is 0 . In this case, the energy of the stained source wavefield is completely lost. In addition, it is unnecessary to use the closed line $l$ as the stained area $\left(D_{s}=l\right)$ (see Fig. 1(b)) because of the propagation of one-way wave. Therefore, we can only choose the stained region above the target structure. When we choose $l_{1}$ as the stained area $\left(D_{s}=l_{1}\right)$ (see Fig. 1(b)), according to Eqs. (2.7) and (2.9), the stained and the real source wavefields inside the surface $\alpha$ are exactly the same, i.e., $\widetilde{u}_{s}\left(x_{m}, z_{m}, t\right)=u_{s}\left(x_{m}, z_{m}, t\right)$, which means that the effective signal associated with the target structure and noise are completely recovered, in which case the SNR cannot be improved. 
a)

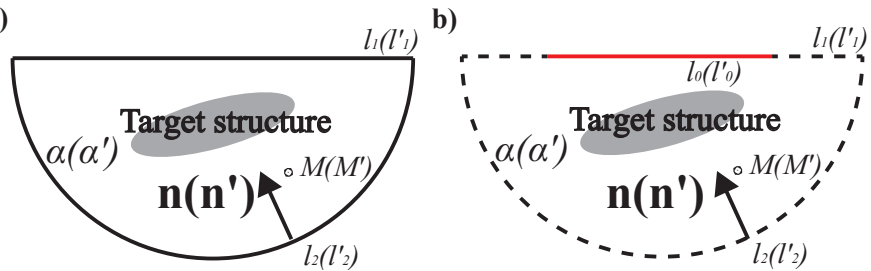

Figure 1: Kirchhoff integral diagrams of the real and the stained source or receiver wavefields: (a) Kirchhoff integral diagram of the real source or receiver wavefield; (b) Kirchhoff integral diagram of the stained source or receiver wavefield.

If we choose $l_{0}$ as the stained area $\left(D_{s}=l_{0}\right)$ (see Fig. 1(b)), according to Eqs. (2.7) and (2.9), we can get the stained source wavefield $\widetilde{u}_{s}\left(x_{m}, \omega\right)$, written as

$$
\widetilde{u}_{s}\left(x_{m}, \omega\right)=\int_{l_{0}}\left[\frac{\partial G\left(x, x_{m}, \omega\right)}{\partial n} u_{s}(x, \omega)-G\left(x, x_{m}, \omega\right) \frac{\partial u_{s}(x, \omega)}{\partial n}\right] d l_{0} .
$$

In this case, the stained source wavefield is different from the real source wavefield. Since the stained area covers the whole target structure horizontally, the energy of the stained source wavefield associated with the target structure is almost completely restored, whereas the noise irrelevant to the target structure is largely removed or suppressed, and, therefore, the SNR of the structure can be improved.

GSP as a typical one-way method was employed in this study to better explain this staining OWEM method. According to Eq. (2.4), we obtain the stained wavefield $\widetilde{u_{s}}(x, z)$, given by

$$
\widetilde{u}_{s}\left(x, z_{k}+\Delta z\right)=\left(1+i \Delta z \frac{A}{k_{0}} \frac{\partial^{2}}{\partial x^{2}}\right) \cdot F^{-1} e^{i \Delta z k_{z}} F e^{-i \Delta z k_{0} \frac{\delta v\left(x, z_{k}\right)}{v\left(x, z_{k}\right)}} \widetilde{u_{s}}\left(x, z_{k}\right),
$$

and the boundary condition is the same as that in Eq. (2.7). The forward-extrapolated stained and the real source wavefields are cross-correlated with the backward-extrapolated receiver wavefield, respectively, and we obtain the source-stained and the real migration images. The zero-lag cross-correlation imaging condition is used for imaging [8], written as

$$
\begin{aligned}
& I(x, z)=\sum_{\omega} u_{s}(x, z, \omega) \cdot u_{r}(x, z, \omega), \\
& \widetilde{I}_{S}(x, z)=\sum_{\omega} \widetilde{u_{s}}(x, z, \omega) \cdot u_{r}(x, z, \omega),
\end{aligned}
$$

where $I(x, z)$ refers to the real image and $\widetilde{I}_{S}(x, z)$ refers to the source-stained image.

\subsection{Staining strategies for OWEM}

We extend the traditional stained source wavefield and construct a new stained receiver wavefield that is relevant to the target structures. The stained receiver wavefield backward propagates synchronously with the real receiver wavefield, like the stained source 
wavefield forward propagating synchronously with the real source wavefield in the same velocity model. The stained receiver wavefield $\widetilde{u}_{r}(x, z)$ is written as

$$
\left(\frac{\partial}{\partial z}-i \sqrt{k_{0}^{2} n^{2}+\frac{\partial^{2}}{\partial x^{2}}}\right) \widetilde{u}_{r}(x, z)=0
$$

and the boundary condition of Eq. (2.14) is given by

$$
\left.\widetilde{u}_{r}(x, z)\right|_{D_{s}(x, z)=1}=u_{r}(x, z),
$$

where $\widetilde{u_{r}}(x, z)$ denotes the stained receiver wavefield. The stained receiver wavefield is only associated with the real receiver wavefield at the stained area (i.e., Eq. (2.15)).

Like in the forward-extrapolated source wavefield, we extrapolate the receiver wavefield backward with the Kirchhoff integral formula. The backward-extrapolated real receiver wavefield $u_{r}\left(x_{m}^{\prime}, \omega\right)$ of point $M^{\prime}\left(x_{m}^{\prime}\right)$ inside the surface $\alpha^{\prime}$ (see Fig. 1(a)) has the form of (Berkhout and Wapenaar., 1989)

$$
u_{r}\left(x_{m}^{\prime}, \omega\right)=\int_{l^{\prime}=l_{1}^{\prime}+l_{2}^{\prime}}\left[\frac{\partial G\left(x^{\prime}, x_{m}^{\prime}, \omega\right)}{\partial n^{\prime}} u_{r}\left(x^{\prime}, \omega\right)-G\left(x^{\prime}, x_{m}^{\prime}, \omega\right) \frac{\partial u_{r}\left(x^{\prime}, \omega\right)}{\partial n^{\prime}}\right] d l^{\prime},
$$

where $l^{\prime}$ represents a closed line that contains the real receiver wavefield within the surface $\alpha^{\prime}$, and $\boldsymbol{n}^{\prime}$ represents the normal of the $l^{\prime}$ line (see Fig. 1(a)). We assume that $x^{\prime}$ goes to infinity along the line $l_{1}^{\prime}$, the integral along the $l_{2}^{\prime}$ in Eq. (2.16) can be ignored.

The propagation of the stained receiver wavefield is a process of reconstructing and tracing the target-oriented receiver wavefield, and for this stained wavefield, the stained area is chosen in the same way as that for the stained source wavefield discussed in Section 2.2. We choose $l_{0}^{\prime}$ as the stained area $\left(D_{s}=l_{0}^{\prime}\right)$ (see Fig. 1(b)) and obtain the stained receiver wavefield ${\widetilde{u_{r}}}_{r}\left(x_{m}^{\prime}, \omega\right)$ of $M^{\prime}$, given by

$$
\widetilde{u}_{r}\left(x_{m}^{\prime}, \omega\right)=\int_{l_{0}^{\prime}}\left[\frac{\partial G\left(x^{\prime}, x_{m}^{\prime}, \omega\right)}{\partial n^{\prime}} u_{r}\left(x^{\prime}, \omega\right)-G\left(x^{\prime}, x_{m}^{\prime}, \omega\right) \frac{\partial u_{r}\left(x^{\prime}, \omega\right)}{\partial n^{\prime}}\right] d l_{0}^{\prime} .
$$

In this scenario, the stained receiver wavefield not only keeps the information of the target structure in amplitude and phase, but also removes a huge amount of noise unrelated to the target structure. Hence, it is important to use the stained receiver wavefield for subsalt imaging to improve the SNR of the weakly illuminated structures.

In this research paper, we propose two new staining strategies for OWEM by using the stained receiver wavefield and use the GSP to explain these new staining strategies. The stained receiver wavefield $\widetilde{u}_{r}(x, z)$ based on the GSP satisfies the following equation:

$$
\widetilde{u}_{r}\left(x, z_{k}+\Delta z\right)=\left(1-i \Delta z \frac{A}{k_{0}} \frac{\partial^{2}}{\partial x^{2}}\right) \cdot F^{-1} e^{i \Delta z k_{z}} F e^{-i \Delta z k_{0} \frac{\delta v\left(x, z_{k}\right)}{v\left(x, z_{k}\right)}} \widetilde{u_{r}}\left(x, z_{k}\right) .
$$

Fig. 2(a) shows the workflow diagram of the first new strategy called receiver-stained strategy for OWEM. In this strategy, we use the real source and the stained receiver wavefields for imaging by applying the imaging condition, given by

$$
\widetilde{I}_{r}(x, z)=\sum_{\omega} u_{s}(x, z, \omega) \cdot \widetilde{u}_{r}(x, z, \omega),
$$



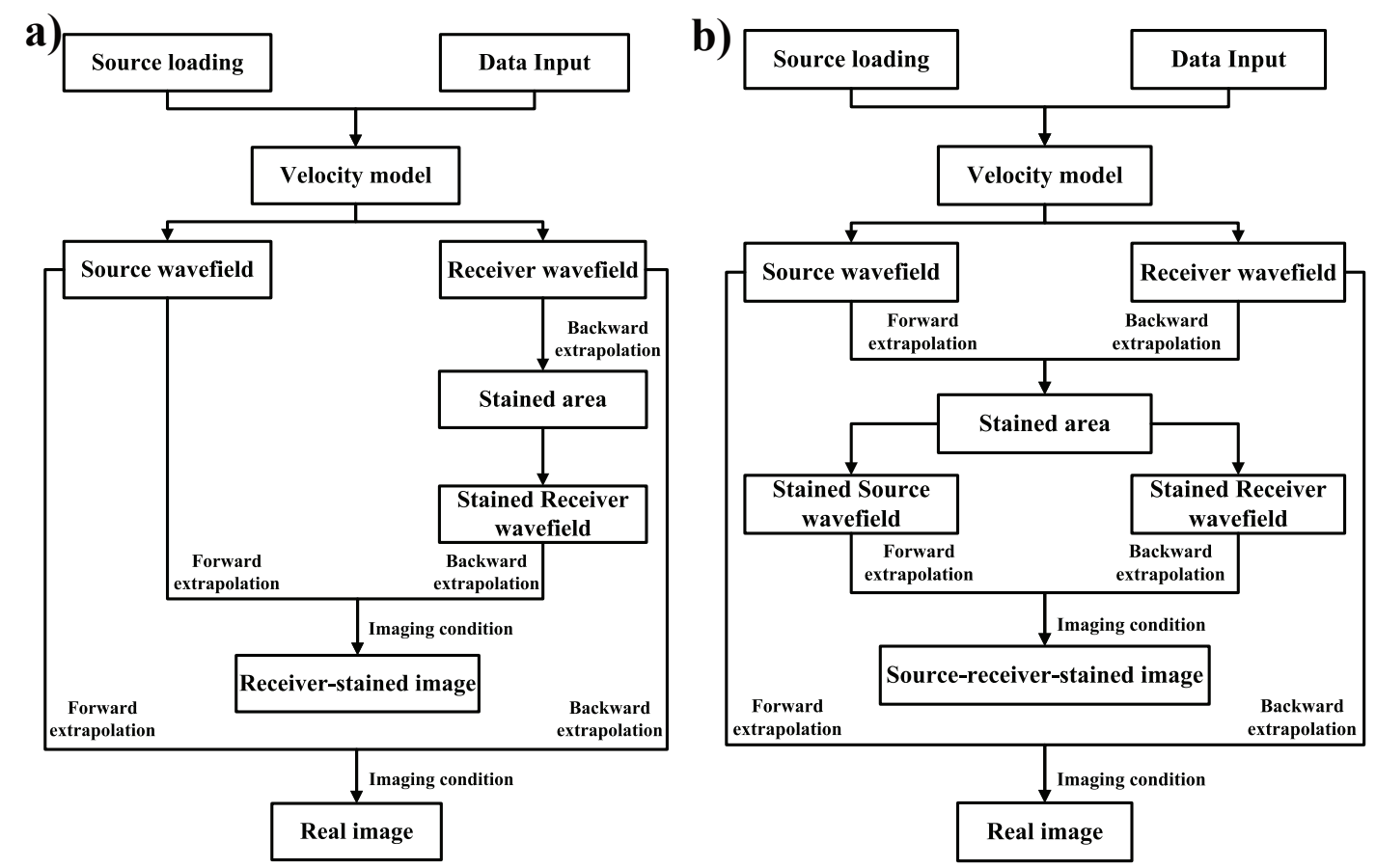

Figure 2: Workflow diagrams of the receiver-stained and the source-receiver-stained strategies. (a) Workflow diagram of the receiver-stained strategy, and (b) workflow diagram of the source-receiver-stained strategy.

where $\widetilde{I}_{r}(x, z)$ denotes the receiver-stained image. The receiver-stained strategy and the source-stained strategy (i.e., conventional staining algorithm) use different stained wavefields for imaging. The source-stained strategy uses the stained source wavefield, which is obtained by tracing and reconstructing the target-oriented source wavefield for imaging, whereas our strategy applies the stained receiver wavefield, which traces and reconstructs the target-oriented receiver wavefield for imaging.

Fig. 2(b) shows the workflow diagram of the second new strategy called sourcereceiver-stained strategy for OWEM and shows that both the source and the receiver wavefields are stained at the same stained area, and the imaging condition is applied to the stained source and recorded wavefields, which is characterised by

$$
\widetilde{I_{s r}}(x, z)=\sum_{\omega} \widetilde{u_{s}}(x, z, \omega) \cdot \widetilde{u_{r}}(x, z, \omega),
$$

where $\widetilde{I_{s r}}(x, z)$ represents the source-receiver-stained image. In fact, the source-receiverstained strategy has a similarity with the survey sinking technology. In the sourcereceiver-stained strategy, the stained wavefields in the stained area are generated as if arranging sources and geophones in this area, similar to the downward-extrapolated wavefields of each depth in survey sinking technology [9]. There are some differences between the survey sinking and the source-receiver-stained technologies. In survey sinking technology, virtual source and receiver are distributed usually throughout a horizontal layer 
of the model, whereas in our source-receiver-stained strategy, the stained area can have an arbitrary shape. The stained wavefield is generated by the injection of the globally propagated real wavefield, while in survey sinking technology, the original wavefield propagates locally (e.g., in the shallow area) to yield the virtual wavefields [49].

Among these staining strategies, the source- and the receiver-stained strategies for OWEM have the effect of unidirectionally tracing and illuminating the seismic wavefields of the target structure, while the source-receiver-stained strategy for OWEM is a process of bidirectionally tracing and illuminating the wavefields. Thus, the sourcereceiver-stained strategy can effectively suppress the noise and highlight the information of the target structure, which is expected to have a better performance in improving the SNR of the weakly illuminated structures such as those in the subsalt.

\section{Numerical examples}

In this section, we discuss several examples that we designed to demonstrate the effectiveness of the staining strategies as applied to the OWEM method. The GSP was used in the following examples.

\subsection{Modelling examples}

To determine the principles on how to choose the stained areas for generating highquality images, we designed the following numerical examples to analyse the influence of different stained areas on improving the SNR of staining wavefields.

\subsubsection{Four-layer model}

To illustrate the infeasibility of the stained areas with infinite horizontal length for improving the quality of images, we design a simple layer velocity model (see Fig. 3(a)). The model size is $1.5 \mathrm{~km} \times 1 \mathrm{~km}$, and the velocities of each layer are $2,3,4$, and $5 \mathrm{~km} / \mathrm{s}$, respectively. The red line in Fig. 3(a) represents the stained area, and it covers the entire horizontal layer of model, including absorbing boundary, similar to the case where $x$ goes to infinity at the stained area. We used the Ricker wavelet as the seismic source with a dominant frequency of $25 \mathrm{~Hz}$ and the shot was located at $0.75 \mathrm{~km}$ on the surface. The shot gathered contained 30 traces, with a trace interval of $5 \mathrm{~m}$. The time sampling interval was $0.5 \mathrm{~ms}$, and the recorded time $T$ was $1 \mathrm{~s}$. Fig. 3(b) and (c) shows the snapshots of the real and the stained source wavefields at $0.4 \mathrm{~s}$, respectively; we can see that the stained source wavefield in Fig. 3(c) contained only the wavefield below the stained area and that its phase remained the same as the real source wavefield. Fig. 3(d) and (e) shows the snapshots of the real and the stained receiver wavefields in the process of backward extrapolation at $T-0.4 s$ [61]. Like in the stained source wavefield, the stained receiver wavefield in Fig. 3(e) consisted only of the back-propagated stained receiver wavefield induced by the stained area, and the phases of the real and the stained receiver wavefields were consistent. 

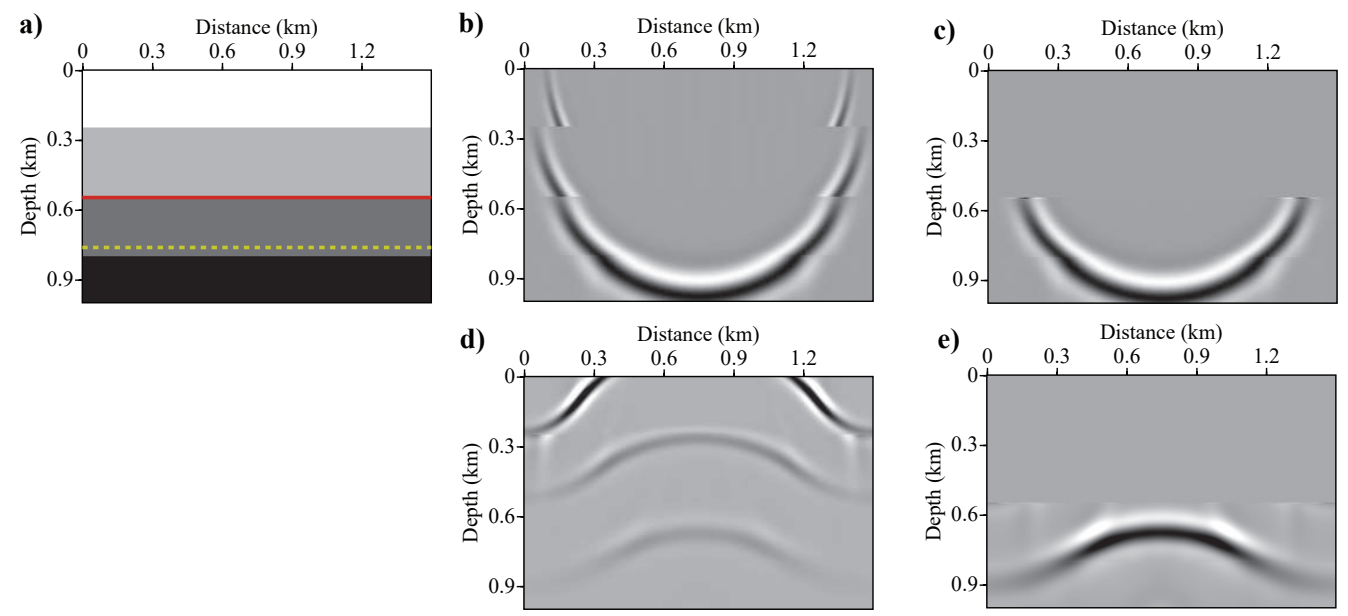

Figure 3: Snapshots of the real and the stained wavefields: (a) the velocity model, (b) the real source wavefield at $0.4 \mathrm{~s},(\mathrm{c})$ the stained source wavefield at $0.4 \mathrm{~s},(\mathrm{~d})$ the real receiver wavefield at $T-0.4 \mathrm{~s}$, and (e) the stained receiver wavefield at $T-0.4 s$.
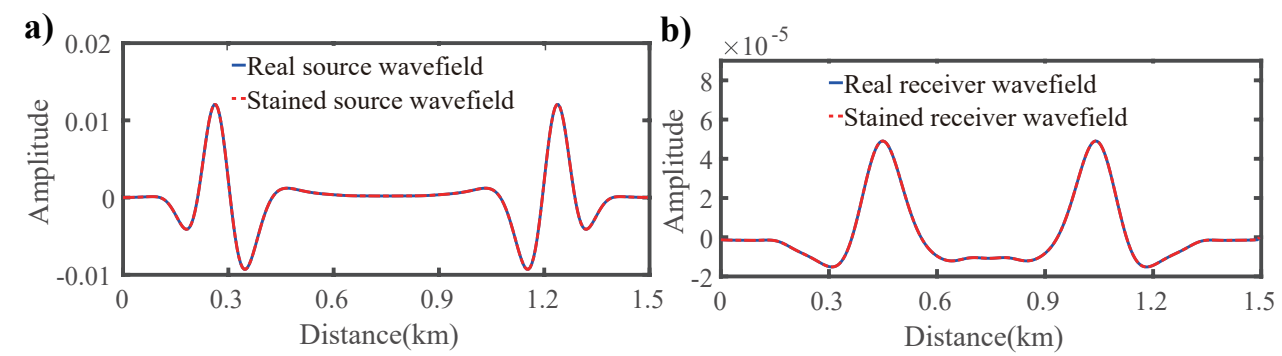

Figure 4: Extractions along the yellow-dashed line in Fig. 3(a) from the various wavefields in Fig. 3: (a) extractions of the real and the stained source wavefields in Fig. 3(b) and (c); (b) extractions of the real and the stained receiver wavefields in Fig. 3(d) and (e).

Fig. 4 shows the extractions along yellow-dashed line (at $z=0.75 \mathrm{~km}$ ) in Fig. 3(a) from the various wavefields in Fig. 3. Fig. 4(a) is the extractions of the real and the stained source wavefields in Fig. 3(b) and (c), we can see that the phase of the stained source wavefield was consistent with the real source wavefield and that the stained source wavefield completely realised fidelity of the amplitude. Fig. 4(b) is the extractions of the real and the stained receiver wavefields in Fig. 3(d) and (e), we can also see that the stained receiver wavefield had phase consistency and amplitude preservation compared to the real receiver wavefield. This example verifies that when we choose the whole horizontal line as the stained area, i.e., $x$ goes to infinity here, the stained wavefields and the real wavefields below the stained area are exactly the same, and SNR will not be improved.

\subsubsection{Three-layer model}

We analysed the stained wavefields in more detail through some experiments to show how to effectively choose the stained areas and thus improve the SNR of the images. We 
design a three-layer velocity model (see Fig. 5(a)). The size of this model is $3.01 \mathrm{~km} \times 2.01$ $\mathrm{km}$, and the grid interval is $10 \mathrm{~m}$. The velocities are $2.5,4.2$, and $3.0 \mathrm{~km} / \mathrm{s}$ in each layer, respectively; a velocity anomaly regarded as the target structure with a length of 0.5 $\mathrm{km}$ lies at a depth of $1.7 \mathrm{~km}$, corresponding to a velocity of $3.3 \mathrm{~km} / \mathrm{s}$. The red line in this model represents the stained area. The data were simulated by the finite difference method, and the Ricker wavelet with a dominant frequency of $15 \mathrm{~Hz}$ served as the seismic source. The shot was located at $1.5 \mathrm{~km}$ on the surface, and the geophones were arranged on the whole surface with a spacing of $10 \mathrm{~m}$. The total time of the data was $2.04 \mathrm{~s}$, and the time sampling interval was $1 \mathrm{~ms}$. We backward-extrapolated the shot gather data with GSP to get the real receiver wavefields of the whole model at any time. We compared the space-time-domain real receiver wavefields (see Fig. 5(b) and (d)) along two horizontal lines (at $z=1.69 \mathrm{~km}$ and $1.5 \mathrm{~km}$, respectively), and find that the yellow-dashed events in Fig. 5(b) and (d) are related to the target structure. Here we refer to the information unrelated to the target structure as 'noise'. According to the location distributions of the yellow events and the 'noise', we chose two stained areas (at $z=1.69 \mathrm{~km}$ with $x \in(1.2$ $\mathrm{km}, 1.8 \mathrm{~km})$ and $z=1.5 \mathrm{~km}$ with $x \in(1.2 \mathrm{~km}, 1.8 \mathrm{~km})$, respectively). The stained receiver wavefields at the two stained areas were extracted from the events inside the red lines in Fig. 5(b) and (d), respectively (see Fig. 5(c) and (e)).

To illustrate the influences of diverse stained areas on signal recovery and noise suppression, we backward-extrapolated the two stained receiver wavefields in Fig. 5(c) and (e), respectively, and recorded the stained wavefields at two different positions (see the yellow-and the gray-inverted triangles in Fig. 5(a)). Fig. 5(f) shows the seismograms of the real and the two stained receiver wavefields at the yellow-inverted triangle position (at $x=1.5 \mathrm{~km}$ with $z=1.7 \mathrm{~km}$, on the target structure) in Fig. 5(a), and these seismograms contain the signal related to the target structure. From this figure, we can see that the phases of the two stained wavefields were almost consistent with the real wavefield. The amplitudes of the two stained wavefields were similar, smaller than that of the real wavefield, which is due to the small horizontal range of the stained areas. Fig. 5(g) shows the seismograms of the three wavefields at the gray-inverted triangle position (at $x=0.5$ $\mathrm{km}$ with $z=1.8 \mathrm{~km}$, outside the target area), and the information of these seismograms was regarded as the 'noise' unrelated to the target structure. Compared to the real wavefield, the two stained wavefields had similar and strong influences on noise suppression. Fig. 5(f) and (g) illustrate that in the stained wavefields, the amount of the recovered signals and the suppressed 'noise' are mainly determined by the horizontal range of the stained area, and are less affected by the depth.

According to the above analysis, we guess that in real situation there is no exact velocity model and target location, and the illumination is poor and SNR is low, we can use the staining strategies. In our staining strategies, the key problem is how to choose an appropriate stained area. These modeling examples demonstrate that the stained area is not necessary to be stick to the target structure, and we only need to ensure that the stained area can cover most energy of the target structure when waves propagate. Both the raytracing technology and the backward-propagated receiver wavefield can help us predict 

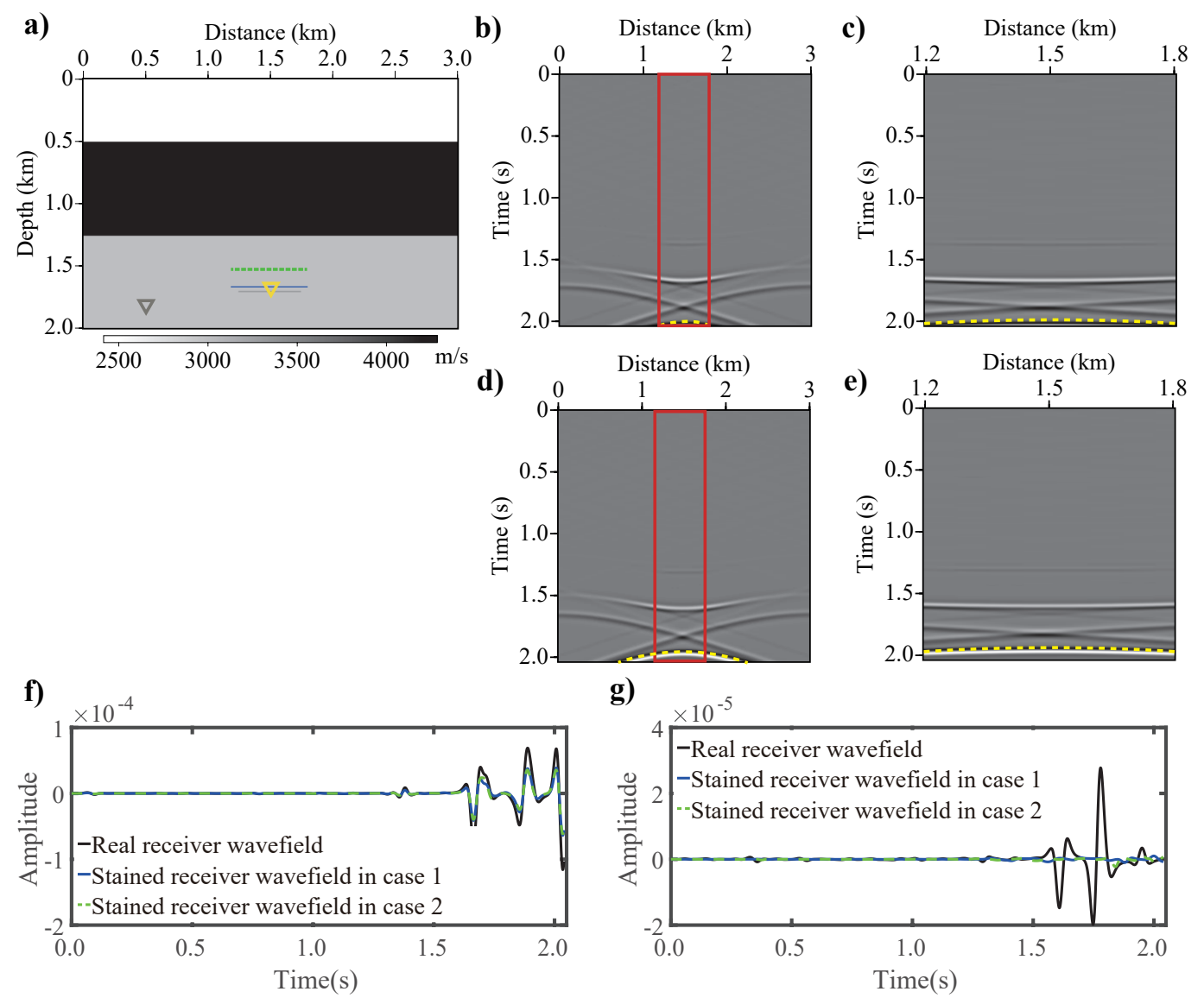

Figure 5: The three-layer velocity model and its space-time-domain real and stained receiver wavefields. (a) The three-layer velocity model, (b) the real receiver wavefield at the depth of $1.69 \mathrm{~km}$, (c) the stained receiver wavefield at the stained area $z=1.69 \mathrm{~km}$ with $x \in(1.2 \mathrm{~km}, 1.8 \mathrm{~km})$ (see the blue line in $(\mathrm{a}))$, (d) the real receiver wavefield at the depth of $1.5 \mathrm{~km}$, (e) the stained receiver wavefield at the stained area $z=1.5 \mathrm{~km}$ with $x \in(1.2 \mathrm{~km}, 1.8 \mathrm{~km})$ (see the green-dashed line in (a)), (f) the seismograms of the real and the stained receiver wavefields at the yellow-inverted triangle position in (a); (c) the seismograms of the real and the stained receiver wavefields at the gray-inverted triangle position in (a); Here case 1 corresponds to the blue stained area in (a), and case 2 corresponds to the green-dashed stained area.

the stained area. Besides, we can also use a multiple staining strategy, i.e. selecting more than one stained area, to solve the problems in real situation.

\subsection{Source-stained strategy for GSP migration}

In this section, we use two models to study the effect of the source-stained strategy for GSP migration and compare the imaging results with those of the conventional GSP and two-way migration methods. The finite-difference RTM in the time domain is used as the two-way migration in this study. 


\subsubsection{Three-layer model}

The design of a three-layer velocity model is shown in Fig. 6(a). This model and its parameters are the same as those in Section 3.1.2. The red line in this model represents the stained area with a horizontal range of $1.2 \mathrm{~km}$ to $1.8 \mathrm{~km}$ and a depth of $1.5 \mathrm{~km}$. The data were simulated in the same way as in Section 3.1.2. The number of shots was 31, and the shot interval was $100 \mathrm{~m}$. The energy of the seismic wave was almost reflected in the upper two layers, whereas a little energy was transmitted to the third layer because of the large impedance contrast at the interface. As a result, the third layer in the model in Fig. 6(a) was a weakly illuminated area, meaning that the velocity anomaly is difficult to identify in seismic imaging. Fig. 6(b) and (c) represent the imaging results of conventional GSP migration and RTM methods, respectively, showing that the images of the velocity anomaly are unrecognised because of the lower resolution and SNR. The source-stained image based on GSP obtained by using Eq. (2.13) shown in Fig. 6(d) was very clear for the target structure and the SNR was greatly improved compared with the conventional GSP and RTM methods.

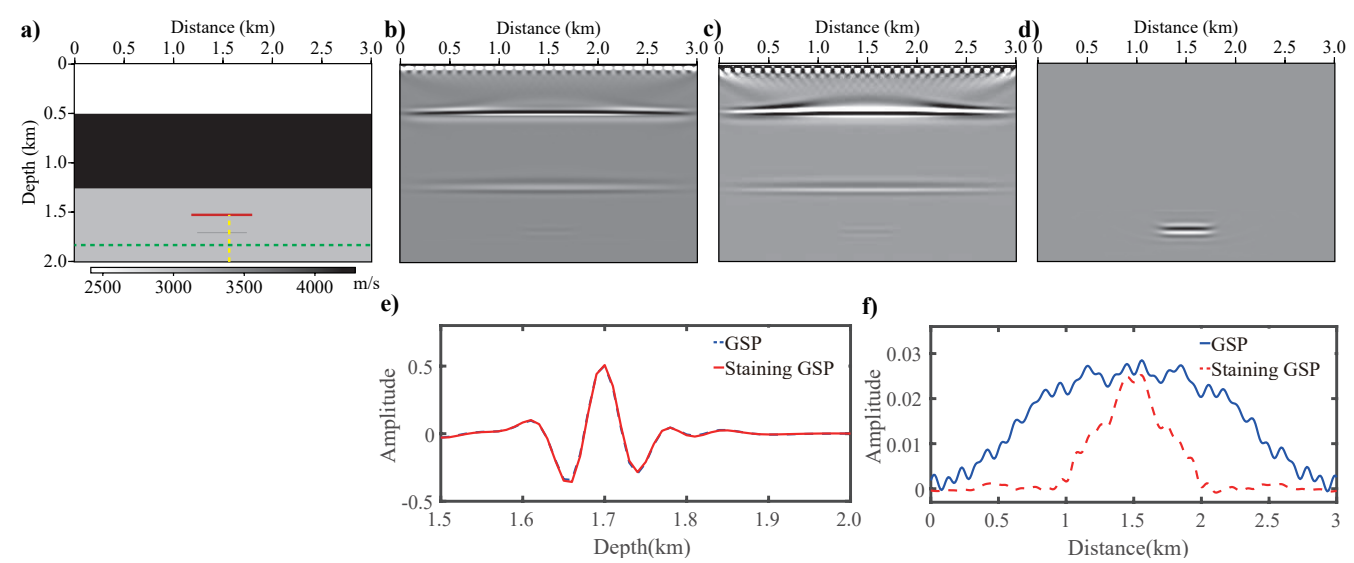

Figure 6: The three-layer velocity model and its imaging results by using different methods. (a) The three-layer velocity model, (b) the imaging result of conventional GSP migration, (c) the imaging result of conventional RTM, (d) the imaging result of staining GSP migration, (e) extractions along the yellow-dashed line in (a) from the images in (b) and (d); (f) extractions along the green-dashed line in (a) from the images in (b) and (d).

To illustrate the restored signal associated with the target structure and the suppressed 'noise' between the staining and conventional GSP methods, we extracted the images in Fig. 6(b) and (d). Fig. 6(e) shows the extractions along the yellow-dashed line (at $x=1.6 \mathrm{~km}$ with $z \in(1.5 \mathrm{~km}, 2 \mathrm{~km})$ ) in Fig. 6(a), we can see that the signal related to the target structure (at $z=1.7 \mathrm{~km}$ ) was almost completely recovered, demonstrating the amplitude fidelity characteristics of the staining method. Fig. 6(f) shows the extractions along the green-dashed line (at $x=1.85 \mathrm{~km}$ ) in Fig. 6(a), we can see that the 'noise' of the source-stained image was significantly lower than that of the conventional GSP migration. This simple example verifies that the staining method can get a high-illumination and high-SNR image. 


\subsubsection{Two-dimensional profile (line A-A') of the SEG/EAGE salt model}

To further verify the staining method, we tested a 2D profile (line A-A') of the Society of Exploration Geophysicists (SEG)/European Association of Geoscientists and Engineers (EAGE) salt model shown in Fig. 7(a). The model size is $1073 \mathrm{~m} \times 228.6 \mathrm{~m}$, and the grid spacing is $7.62 \mathrm{~m}$. We used the Ricker wavelet as the seismic source with a dominant frequency of $13 \mathrm{~Hz}$, and the shot was located on the surface. The number of shots was 28 , and each shot gathered contained 140 traces, with a trace interval of $7.62 \mathrm{~m}$. The time sampling interval was $0.8 \mathrm{~ms}$, and the recorded time was $8.0 \mathrm{~s}$. We selected the red line as stained area 1 and the blue line as stained area 2, and the sediments close to the bottom of the two stained areas were regarded as the target structures 1 and 2, respectively. The imaging results of conventional GSP migration and RTM are shown in Fig. 7(b) and (c), respectively, and those images at the target areas were blurred as a result of the salt dome. Fig. 7(d) and (e) shows the imaging results corresponding to stained areas 1 and 2 of the staining GSP method, respectively; the two images of target structures 1 and 2 were much better than the imaging results of conventional GSP and RTM methods.

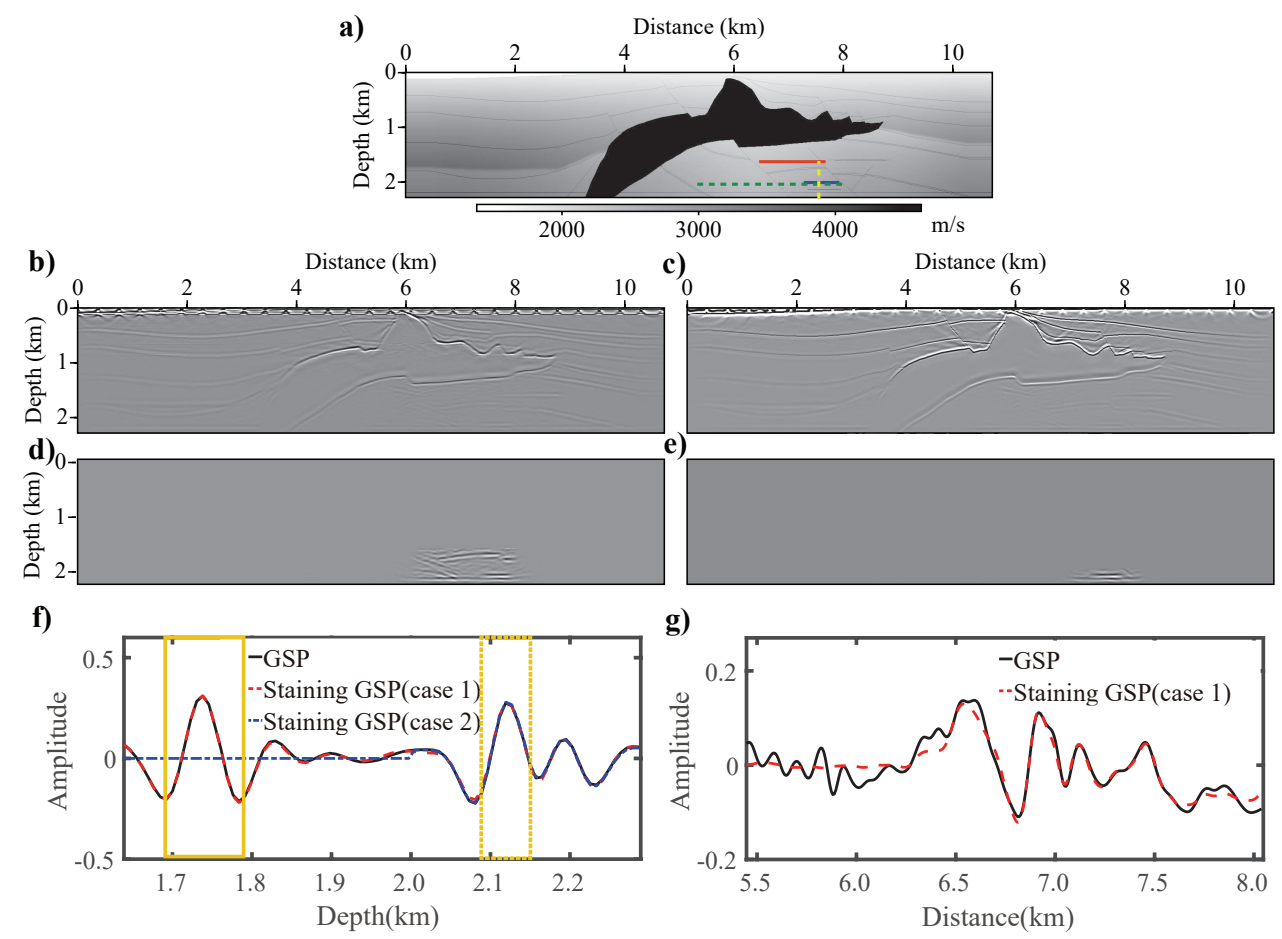

Figure 7: Two-dimensional profile (line A-A') of the SEG/EAGE salt model and its imaging results by using different methods. (a) The velocity model, (b) the imaging result of conventional GSP, (c) the imaging result of conventional RTM, (d) the imaging result of staining GSP corresponding to the red stained area in (a); (e) the imaging result of staining GSP corresponding to the blue stained area in (a); (f) extractions along the yellow-dashed line in (a) from the images in (b), (d) and (e); (g) extractions along the green-dashed line in (a) from the images in (b) and (d). 
We extracted the images obtained by the conventional and the staining GSP migration methods. Fig. 7(f) shows the extractions along the yellow-dashed line (at $x=7.54 \mathrm{~km}$ with $z \in(1.64 \mathrm{~km}, 2.28 \mathrm{~km}))$ in Fig. 7(a), we can see that the two source-stained images and the image of conventional GSP migration agreed well on the target structures (see the events inside of the yellow and yellow-dashed lines in Fig. 7(f)), demonstrating the effectiveness of the source-stained strategy for GSP migration on signal preservation compared to the conventional GSP migration. Fig. 7(g) shows the extractions along the green-dashed line (at $z=2.06 \mathrm{~km}$ with $x \in(5.3 \mathrm{~km}, 7.9 \mathrm{~km})$ ) in Fig. 7(a) from the images in Fig. 7(b) and (d), illustrating that the 'noise' unrelated to the target structure 1 was suppressed in the source-stained image.

The numerical experiments in Sections 3.2.1 and 3.2.2 demonstrate that the sourcestained strategy for GSP migration can effectively improve the SNR of the poorly illuminated structure such as the subsalt in comparison with the conventional GSP method and RTM method.

\subsection{Receiver-stained and source-receiver-stained strategies for GSP migration}

In this section, we also chose the 2D profile (line A-A') of the SEG/EAGE salt model shown in Fig. 8(a), and the red line in Fig. 8(a) was used as the stained area, and the sediments close to the bottom of the stained area were regarded as the target structure. The data were simulated in the same way as in Section 3.2.2. Fig. 8(b) shows the source-stained image by employing the source-stained strategy for GSP migration (see Eq. (2.13)). The receiver-stained image obtained by the receiver-stained strategy (see Eq. (2.19)), which uses the stained receiver wavefield for GSP migration, is shown in Fig. 8(c), and the improvement is not obvious compared with the source-stained strategy. Fig. 8(d) shows the source-receiver-stained image obtained by the source-receiverstained strategy (see Eq. (2.20)), which uses both the stained source and receiver wavefields for GSP migration. In comparison with the source- and the receiver-stained images, the 'noise' of the source-receiver-stained image was largely suppressed, especially in the regions away from the target area.

We extracted the three images in Fig. 8 to compare the three staining strategies in detail. Fig. 8(e) shows the extractions along the yellow-dashed line (at $x=6 \mathrm{~km}$ with $z \in(1.45 \mathrm{~km}, 2.06 \mathrm{~km}))$ in Fig. 8(a). Compared to the source- and the receiver-stained images, the source-receiver-stained image had slightly distorted signal (see the events inside the yellow line in Fig. 8(e)), but its noise was largely suppressed. Fig. 8(f) shows the extractions along the green-dashed line (at $z=2.02 \mathrm{~km}$ with $x \in(4.3 \mathrm{~km}, 7.8 \mathrm{~km})$ ) in Fig. 8(a), illustrating that the source-receiver-stained strategy had a better effect on the 'noise' suppression than the other staining strategies.

The results of the numerical example in this section show that the different staining strategies for GSP migration can improve the SNR of the subsalt structures. The sourcereceiver-stained strategy played a significant role in bidirectionally suppressing noise, and the SNR was higher than that obtained by the source-and the receiver-stained strate- 

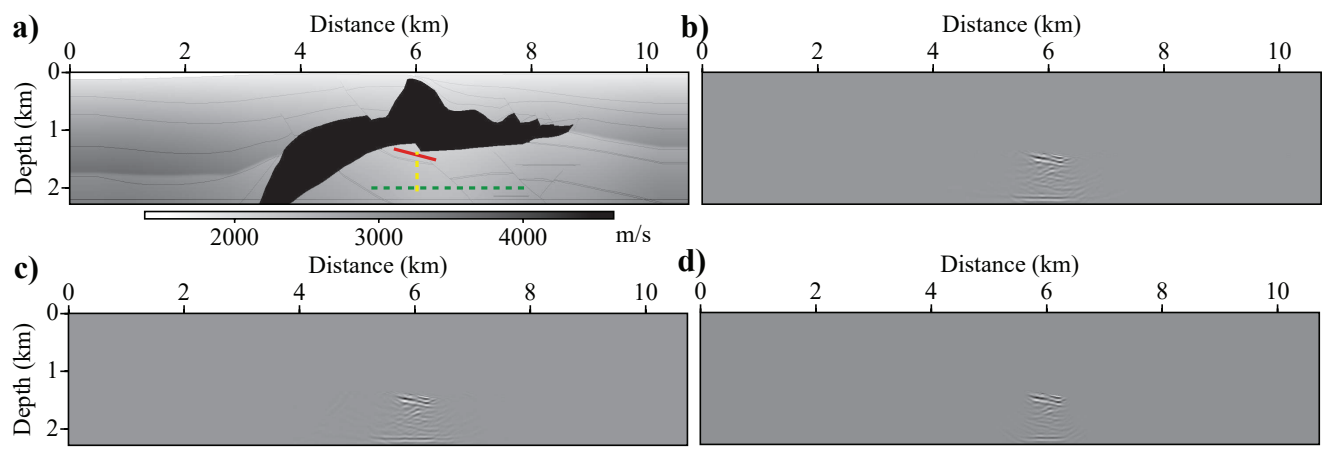

e)

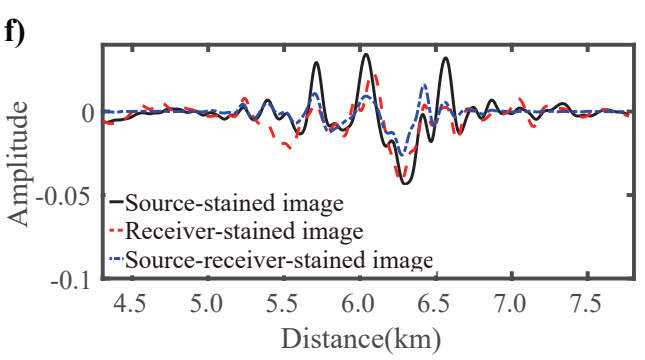

Figure 8: Two-dimensional profile (line A-A) of the SEG/EAGE salt model and its imaging results by using different staining strategies for GSP. (a) The velocity model, (b) the image obtained by the source-stained strategy for GSP, (c) the image obtained by the receiver-stained strategy for GSP, (d) the image obtained by the source-receiver-stained strategy for GSP, (e) the images extracted along the yellow-dashed line in (a); ( $f$ ) the images extracted along the green-dashed line in (a).

gies for GSP migration. These staining strategies are expected to be extended to other seismic modelling and migration methods.

\section{Conclusion}

We demonstrated the reliability and effectiveness of staining GSP based on OWEM methods for poorly illuminated structures in subsalt zone imaging; this staining GSP method can improve the illumination of subsalt areas and obtain higher SNR images of the target structure compared to the conventional one-way and two-way methods. In addition, we proposed a stained receiver wavefield, which is a receiver wavefield that is relevant to the target structure, and the stained receiver wavefield at the stained area as the boundary condition backward extrapolates synchronously with the real receiver wavefield, like the regular stained source wavefield forward extrapolating synchronously with the real source wavefield. Both the stained source and receiver wavefields are target-oriented to trace the information related to the target structure. The staining algorithm was illustrated in the experiments on stained wavefield modelling and provides two new strategies for subsalt imaging. One strategy is to cross-correlate the real source wavefield with the stained receiver wavefield, and the other is to carry out the cross-correlation on the 
stained source and receiver wavefields, and we obtain two stained images of the target structures. Numerical experiments verified that the source-receiver-stained strategy, which has the effects of bi-directionally tracing and illuminating the information of the wavefield in target areas, can obtain higher SNR images compared to the conventional staining algorithms for the GSP and the receiver-stained strategy. In theory, these staining strategies are applicable to any migration methods and we recommend the sourcereceiver-stained strategy for imaging.

\section{Acknowledgments}

The authors gratefully acknowledge Dr. Lianjie Huang, Dr. Michael Fehler and Corinna $\mathrm{Yu}$ for their help with this paper. Our deep gratitude goes to the two anonymous reviewers for their careful work and thoughtful suggestions that have helped improve this paper substantially. The authors would also like to thank Quanli Li, Wenyang Chen, Qihua Li and Lu Yang for their fruitful discussions. This study received support from the National Key Research and Development Program of China (No. 2017YFB0202903) and the National Natural Science Foundation of China (41774121).

\section{References}

[1] A. J. Berkhout, Steep dip finite-difference migration, Geophys. Prospect., 27(1979), 196-213.

[2] A. J. Berkhout and C. Wapenaar, One-way versions of the Kirchhoff integral, Geophysics, 54(1989), 460-467.

[3] T. Burch, B. Hornby, H. Sugianto, and B. Nolte, Subsalt 3D VSP imaging at deimos field in the deepwater gulf of mexico, The Leading Edge, 29(2010), 680-685.

[4] J. Cao and R. S. Wu, Fast acquisition aperture correction in prestack depth migration using beamlet decomposition, Geophysics, 74(2009), S67-S74.

[5] B. Chen, X. F. Jia, and X. B. Xie, Broadband seismic illumination and resolution analyses based on staining algorithm, Appl. Geophys., 13(2016), 480-490.

[6] B. Chen and X. F. Jia, Staining algorithm for seismic modeling and migration, Geophysics, 79(2014), S121-S129.

[7] J. B. Chen and H. Liu, Two kinds of separable approximations for the one-way wave operator, Geophysics, 71(2005), T1-T5.

[8] J. F. Claerbout, Toward a unified theory of reflector mapping, Geophysics, 36(1791), 467-481.

[9] J. F. Claerbout, Imaging the earth's interior, Blackwell scientific publications Oxford, 1(1985).

[10] L. Dale and J. M. Slack, Fate map for the 32-cell stage of xenopus laevis, Development, 99(1987), 527-551.

[11] M. V. de Hoop, B. Ursin, and A. E. Malcolm, Subsalt imaging with internal multiples, Expanded Abstr., 78th Ann. Internat. SEG Mtg., 2008, 2461-2465.

[12] J. Etgen, S. H. Gray, and Y. Zhang, An overview of depth imaging in exploration geophysics, Geophysics, 74(2009), WCA5-WCA17.

[13] J. Gazdag, Wave equation migration with the phase-shift method, Geophysics, 43(1978), 1342-1351. 
[14] J. Gazdag and P. Sguazzero, Migration of seismic data by phase shift plus interpolation, Geophysics, 49(1984), 124-131.

[15] F. Ginhoux, M. Greter, M. Leboeuf, S. Nandi, P. See, S. Gokhan, M. F Mehler, S. J. Conway, L. G. Ng, E. R. Stanley, I. M. Samokhvalov, and M. Merad, Fate mapping analysis reveals that adult microglia derive from primitive macrophages, Science, 330(2010), 841-845.

[16] G. Q. Zhang, S. L. Zhang, Y. X. Wang, and C. Y. Liu, A new algorithm for finite-difference migration of steep dips, Geophysics, 53(1988), 167-175.

[17] A. Guitton, Shot-profile migration of multiple reflections, Expanded Abstr., 72nd Ann. Internat. SEG Mtg., 2002, 1296-1299.

[18] D. Hale, N. R. Hill, and J. Stefani, Imaging salt with turning seismic waves, Geophysics, 57(1991), 1453-1462.

[19] J. Y. Hu and X. F. Jia, Numerical modeling of seismic waves using frequency-adaptive meshes, J. Appl. Geophys., 131(2016), 41-53.

[20] L. J. Huang and C. M. Fehler, Quasi-born fourier migration, Geophys. J. Int., 140(2000), 521-534.

[21] L. J. Huang and R. S. Wu, Prestack depth migration with acoustic screen propagators, Expanded Abstr., 66th Ann. Internat. SEG Mtg., 1996, 415-418.

[22] L. J. Huang, M. C. Fehler, P. M. Roberts, and C. C. Burch, Extended local rytov fourier migration method, Geophysics, 64(1999), 1535-1545.

[23] M. P. A. Jackson, B. C. Vendeville, and D. D. Schultz-Ela, Salt-related structures in the gulf of mexico: A field guide for geophysicists, The Leading Edge, 13(1994), 837-842.

[24] S. Ji, T. Huang, K. Fu, and Z. X. Li, Dirty salt velocity inversion: The road to a clearer subsalt image, Geophysics, 76(2011), WB169-WB174.

[25] X. F. Jia and R. S. Wu, Superwide-angle one-way wave propagator and its application in imaging steep salt flanks, Geophysics, 74(2009), S75-S83.

[26] X. F. Jia and L. Yang, A memory-efficient staining algorithm in 3D seismic modelling and imaging, J. Appl. Geophys., 143(2017), 62-73.

[27] S. W. Jin, R. S. Wu, and C. B. Peng, Prestack depth migration using a hybrid pseudo-screen propagator, Expanded Abstr., 68th Ann. Internat. SEG Mtg., 1998, 1819-1822.

[28] S. W. Jin, R. S. Wu, and C. B. Peng, Seismic depth migration with pseudo-screen propagator, Computat. Geosci., 3(1999), 321-335.

[29] W. Kessinger and M. Ramaswamy. Subsalt imaging using mode converted energy and acoustic depth migration, Expanded Abstr., 66th Ann. Internat. SEG Mtg., 1996, 566-569.

[30] J. M. H. Le Rousseau and M. V. de Hoop, Scalar generalized-screen algorithms in transversely isotropic media with a vertical symmetry axis, Geophysics, 66(2001), 1538-1550.

[31] M. W. Lee and S. Y. Suh, Optimization of one-way wave equations, Geophysics, 50(1985), 1634-1637.

[32] J. P. Leveille, I. F. Jones, Z. Z. Zhou, B. Wang, and F. Q. Liu, Subsalt imaging for exploration, production, and development: A review, Geophysics, 76(2011), WB3-WB20.

[33] Y. Z. Lin and L. J. Huang, Building subsurface velocity models with sharp interfaces using interface-guided seismic full-waveform inversion, Pure Appl. Geophys., 174(2017), 40354055.

[34] Q. H. Li and X. F. Jia, Generalized staining algorithm for seismic modeling and migration, Geophysics, 82(2016), T17-T26.

[35] Y. K. Liu, X. Chang, D.G. Jin, R.Q. He, H. C. Sun, and Y. C. Zheng, Reverse time migration of multiples for subsalt imaging, Geophysics, 76(2011), WB209-WB216.

[36] R. S. Lu, D. E. Willen, and I. A. Watson, Identifying, removing, and imaging ps conversions 
at salt-sediment interfaces, Geophysics, 68(2003), 1052-1059.

[37] V. Maarten, J. H. Le Rousseau, and R. S. Wu, Generalization of the phase-screen approximation for the scattering of acoustic waves, Wave Motion, 31(2000), 43-70.

[38] G. F. Margrave and R. J. Ferguson, Wavefield extrapolation by nonstationary phase shift, Geophysics, 64(1999), 1067-1078.

[39] D. Muerdter and D. Ratcliff, Understanding subsalt illumination through ray-trace modeling, part 1: Simple 2-d salt models, The Leading Edge, 20(2001), 578-594.

[40] M. J. O'Brien and S. H. Gray, Can we image beneath salt?, The Leading Edge, 15(1996), 17-22.

[41] G. W. Purnell, Imaging beneath a high-velocity layer using converted waves, Geophysics, 57(1992), 1444-1452.

[42] D. Ristow and T. Rühl, Fourier finite-difference migration, Geophysics, 59(1994), 1882-1893.

[43] H. Shen, S. Mothi, and U. Albertin, Improving subsalt imaging with illumination-based weighting of RTM 3D angle gathers, Expanded Abstr., 81st Ann. Internat. SEG Mtg., 2011, 3206-3211.

[44] Y. Shi and Y. H. Wang, Reverse time migration of 3D vertical seismic profile data, Geophysics, 81(2015), S31-S38.

[45] P. L. Stoffa, J. T. Fokkema, R. M. de Luna Freire, and W. P. Kessinger, Split-step fourier migration, Geophysics, 55(1990), 410-421.

[46] R. H. Stolt, Migration by fourier transform, Geophysics, 43(1978), 23-48.

[47] Y. X. Tang and B. Biondi, Target-oriented wavefield tomography using synthesized born data, Geophysics, 76(2010), WB191-WB207.

[48] B. Wang, Y. Kim, C. Mason, and X. S. Zeng, Advances in velocity model-building technology for subsalt imaging, Geophysics, 73(2008), VE173-VE181.

[49] B. Y. Wu, R. S. Wu, and J. H. Gao, Dreamlet source-receiver survey sinking prestack depth migration, Geophysical Prospecting, 61(2013), 63-74.

[50] R. S. Wu, Wave propagation, scattering and imaging using dual-domain one-way and onereturn propagators, Pure Appl. Geophys., 160(2003), 509-539.

[51] R. S. Wu, Wide-angle elastic wave one-way propagation in heterogeneous media and an elastic wave complex-screen method, J. Geophys. Res.: Sol. Ea., 99(1994), 751-766.

[52] R. S. Wu, Synthetic seismograms in heterogeneous media by one-return approximation, Pure Appl. Geophys., 148(1996), 155-173.

[53] R. S. Wu, H. M. Guan, and X. Y. Wu, Imaging steep sub-salt structures using converted wave paths, Expanded Abstr., 71st Ann. Internat. SEG Mtg., 2001, 845-848.

[54] R. S. Wu and L. J. Huang, Reflected wave modeling in heterogeneous acoustic media using the de wolf approximation, Mathematical Methods in Geophysical Imaging III, SPIE 2571(1995), 176-186.

[55] R. S. Wu and M. Q. Luo, Comparison of different schemes of image amplitude correction in prestack depth migration, Expanded Abstr., 75th Ann. Internat. SEG Mtg., 2005, 2060-2063.

[56] R. S. Wu, M. Luo, S. C. Chen, and X. B. Xie, Acquisition aperture correction in angle-domain and true-amplitude imaging for wave equation migration, Expanded Abstr., 74th Ann. Internat. SEG Mtg., 2004, 937-940.

[57] R. S. Wu and V. Maarten, Accuracy analysis and numerical tests of screen propagators for wave extrapolation, Mathematical Methods in Geophysical Imaging IV, SPIE, 2822(1996), 196-209.

[58] R. S. Wu, R. Yan, X. B. Xie, and D. Walraven, Elastic converted-wave path migration for subsalt imaging, Expanded Abstr., 80th Ann. Internat. SEG Mtg., 2010, 3176-3180. 
[59] X. B. Xie, S. W. Jin, and R.S. Wu, Wave-equation-based seismic illumination analysis, Geophysics, 71(2006), S169-S177.

[60] X. B. Xie and R. S. Wu, Improve the wide angle accuracy of screen method under large contrast, Expanded Abstr., 68th Ann. Internat. SEG Mtg., 1998, 1811-1814.

[61] X. B. Xie and R. S. Wu, A depth migration method based on the full-wave reverse-time calculation and local one-way propagation, Expanded Abstr., 76th Ann. Internat. SEG Mtg., 2006, 2333-2337.

[62] R. Yan, R. S. Wu, X. B. Xie, and D. Walraven, A hybrid elastic one-way propagator for strongcontrast media and its application to subsalt imaginghybrid elastic one-way propagator, Geophysics, 82(2017), S467-S478.

[63] T. N. Yang, J. Shragge, and P. Sava, Illumination compensation for image-domain wavefield tomography, Geophysics, 78(2013), U65-U76.

[64] Y. Zhang, S. Xu, N. Bleistein, and G. Q. Zhang, True-amplitude, angle-domain, commonimage gathers from one-way wave-equation migrations, Geophysics, 72(2007), S49-S58.

[65] Y. Zhang, G. Q. Zhang, and N. Bleistein, Theory of true-amplitude one-way wave equations and true-amplitude common-shot migration, Geophysics, 70(2005), E1-E10. 